\title{
ALGUNOS ANTECEDENTES METEOROLÓGICOS QUE EXPLICAN LAS IRRUPCIONES DE ELASMODERUS WAGENKNECHTI (ORTHOPTERA: TRISTIRIDAE) EN LA REGIÓN DEL SEMIÁRIDO DE CHILE ${ }^{1}$
}

\author{
SOME METEOROLOGICAL EVIDENCE EXPLAINING THE ELASMODERUS \\ WAGENKNECHTI OUTBREAKS (ORTHOPTERA: TRISTIRIDAE) IN THE \\ SEMIARID REGION OF CHILE ${ }^{l}$
}

\author{
Jorge Cepeda-Pizarro ${ }^{2 *}$; Solange Vega ${ }^{2}$; Mario Elgueta ${ }^{3}$; Jaime Pizarro-Araya ${ }^{2}$
}

\begin{abstract}
RESUMEN
En los sectores de interfluvios de la región desértica transicional de Chile (25-32 ${ }^{\circ}$ Lat. S.) existe un conjunto de especies de ortópteros que, bajo ciertas condiciones ambientales, irrumpen demográficamente. Una de estas especies es Elasmoderus wagenknechti (Liebermann) (Orthoptera: Tristiridae), especie endémica y erémica a Chile. El objetivo del trabajo fue documentar, desde un punto de vista meteorológico, los eventos irruptivos (brotes en adelante) ocurridos en el área de Combarbalá $\left(31^{\circ} 10^{\prime} \mathrm{S}, 71^{\circ} 00^{\prime} \mathrm{O}\right)$ en los años 1970, 1996 y 1999. Nuestra hipótesis de trabajo fue que tanto la precipitación como la distribución de la temperatura en los años de ocurrencia del brote pueden jugar un papel en el gatillamiento de ellos. Para documentar esta hipótesis, examinamos los patrones térmico y pluviométrico del año del brote así como aquellos mostrados por los dos años previos al mismo. Análisis de varianza mostraron diferencias en densidad entre sitios del área afectada en un mismo año así como diferencias entre años. Las densidades promedios se estimaron entre 10-50 ind $/ \mathrm{m}^{2}$ en 1970. Estos valores fueron mucho más bajos en 1996 y 1999 $(0,17-0,37$ ind $/ \mathrm{m}^{2}$ y $0,49-0,58 \mathrm{ind} / \mathrm{m}^{2}$, respectivamente), probablemente debido al fuerte control químico aplicado al comienzo de la estación. En relación a nuestra hipótesis, aunque los máximos anuales de temperatura tendieron ligeramente a desplazarse al comienzo de la estación de verano en los años previos al brote, no se observaron desviaciones claras entre esta conducta térmica y el patrón histórico del área. Para los tres eventos examinados, la precipitación anual registrada en el año del evento y aquella del primer año anterior fue inferior al promedio histórico del área (30 años). La conducta de la precipitación del segundo año anterior al brote fue inconsistente. Fue menor en los brotes de los años 1970 y 1996, pero claramente superior en el brote de 1999. La distribución mensual de la precipitación del año del brote mostró diferencias con el patrón mensual histórico. La información meteorológica disponible a la fecha apoya la idea que temperaturas relativamente altas en verano, año con menor precipitación respecto de la media y desviaciones de la distribución de la precipitación mensual respecto de su patrón histórico, pueden gatillar la ocurrencia de brotes de E. wagenknechti. Esta evidencia puede quedar enmascarada como consecuencia del fuerte control químico que se aplica a comienzos de la estación.
\end{abstract}

Palabras clave: Orthoptera, insectos de secano, irrupciones poblacionales, zonas áridas, biometeorología, Chile.

\section{ABSTRACT}

In the rangeland areas of the Chilean transitional desert $\left(25-32^{\circ}\right.$ Lat $\left.S\right)$, there is a group of orthopterous species that, under certain environmental conditions, irrupt demographically. One of these species is Elasmoderus wagenknechti (Liebermann) (Orthoptera: Tristiridae), an endemic and eremic species to Chile. The objective of this work was to document, from a meteorological point of view, the irruptive events (outbreak onward) occurred in some sites of the Combarbala area $\left(31^{\circ} 10^{\prime} \mathrm{S}, 71^{\circ} 00^{\prime} \mathrm{W}\right.$ ) in the years

1 Estudio financiado por la Dirección de Investigación de la Universidad de La Serena, a través de los proyectos DIULS 220.2.7 y DIULS 220.2.17.

2 Laboratorio de Entomología Ecológica, Departamento de Biología, Facultad de Ciencias, Universidad de La Serena, Casilla 599, La Serena, Chile.

E-mail: jcepeda@userena.cl*Autor para correspondencia.

3 Sección Entomología, Museo Nacional de Historia Natural, Casilla 787, Santiago, Chile.

Fecha de Recepción: 05 Junio 2006

Fecha de Aceptación: 17 Julio 2006 
1970, 1996, and 1999. Our working hypothesis was that both rainfall and temperature could have played a role in triggering these outbreaks. To document the hypothesis, we examined the thermal and rainfall patterns of the outbreak year as well as those shown by the 2 previous ones. AOV-tests revealed differences in grasshopper densities among sites for a same outbreak year and between years. Average densities were estimated between 10-50 ind/ $\mathrm{m}^{2}$ in 1970; in 1996 and 1999, in turn, these values were much lower $\left(0,17-0,37 \mathrm{ind} / \mathrm{m}^{2}\right.$ and $0,49-0,58 \mathrm{ind} / \mathrm{m}^{2}$, respectively), probably due to heavy chemical control. Regarding our hypothesis, although the аппиаl maximum values of temperature slightly tended to be reached earlier in the summer season in the years previous to the outbreak, no evident deviations were detected between this thermal behaviour and the historical thermal pattern of the area. Annual rainfall registered during the outbreak years and the year before was lower than the 30-years mean value. The recorded rainfall of the second year before was, however, inconsistent. It was drier in the 1970 and 1996 outbreaks, but moister in the 1999-outbreak. Some deviations were observed between the 30-years monthly historical pattern and the monthly rainfall pattern of the outbreak years. The meteorological data available to date support the idea that relatively high summer temperatures, recorded rainfall lower than the historical mean value, and deviations from the monthly rainfall pattern may induce E. wagenknechti outbreaks. This evidence may be obscured because heavy chemical control applied early in the season.

Key words: Orthoptera, rangeland insects, population outbreaks, arid lands, biometeorology, Chile.

\section{INTRODUCCIÓN}

De las diversas causas que estarían regulando las irrupciones poblacionales de langostas el efecto de factores meteorológicos ha sido analizado por distintos autores (Gage y Mukerji, 1977; Farrow y Longstaff, 1986; Capinera y Horton, 1989; Fielding y Brusven, 1990; Joern y Gaines, 1990; Schell y Lockwood, 1997; Gardner y Thompson, 2001; Corcket et al., 2002; Luker et al., 2002; Amarasekare y Edelson, 2004; Wagner, 2005). De acuerdo a varios de ellos, la importancia de estos factores varía según la latitud (Capinera y Horton, 1989; Joern y Gaines, 1990; Wachter et al., 1998; Berner et al., 2004). Por ejemplo, en Norteamérica, a latitudes medias, la precipitación resultaría más importante que la temperatura; aparentemente las especies se encontrarían más limitadas por la disponibilidad de alimento que por la velocidad de desarrollo de los individuos (Nerney, 1961; Capinera y Horton, 1989; Lockwood y Lockwood, 1991; Vermeire $e t$ $a l .$, 2004). A latitudes altas, la abundancia estaría negativamente relacionada con la precipitación, respuesta aparentemente relacionada a una menor velocidad de desarrollo o a la acción de patógenos (Gage y Mukerji, 1977; Skinner y Child, 2000). Por otro lado, condiciones secas y cálidas favorecerían la abundancia de langostas a latitudes bajas, zonas de montañas, sitios templados o de lluvia estacional (Joern y Gaines, 1990; Kemp y Cigliano, 1994; Bauer et al., 2005). A la fecha, el papel de los factores meteorológicos en la ocurrencia de brotes de langostas chilenas, entre ellos los tristíridos, no ha sido examinado.

Las langostas de la familia Tristiridae (Orthoptera: Acridoidea) constituyen un grupo de insectos que habitan principalmente la zona de transición Sudamericana (Morrone 2006) de Perú, Argentina y Chile (Cigliano, 1989a, 1989b, 1991; Cigliano et al., 1989; Donato, 2006). Dentro de Tristiridae, Elasmoderus es un género endémico y erémico a Chile (Cigliano, 1989a; Roig-Juñent y Flores, 2001; Roig-Juñent et al., 2006). Está constituido por tres especies, destacándose E. wagenknechti (Liebermann) por presentar irrupciones demográficas en zonas interfluviales del desierto transicional de Chile (Moroni, 1972; Toro, 1972; Cepeda-Pizarro et al., 2003). Estas irrupciones tienen una distribución focal en las zonas afectadas, desconociéndose a la fecha las causas de ello (Cepeda-Pizarro et al., en revisión).

En este trabajo analizamos las características térmicas y pluviométricas asociadas a los brotes ocurridos en los años 1970, 1996 y 1999. Nuestra hipótesis fue que las características térmicas y pluviométricas del período previo al año de ocurrencia del brote juegan un papel importante en la ocurrencia de las irrupciones poblacionales de esta especie. El objetivo del trabajo fue documentar y analizar las características térmicas y pluviométricas asociadas a los brotes mencionados.

\section{MATERIALES Y MÉTODOS}

\section{ÁREA DE ESTUDIO}

Los brotes de E. wagenknechti estudiados por nosotros ocurrieron en 1970, 1996 y 1999 en la zona de Combarbalá ( $31^{\circ} 10^{\prime} \mathrm{S}, 71^{\circ} 00^{\prime} \mathrm{W}$, Provincia de Limarí, IV Región, Chile) (Figura 1). Los sitios afectados fueron Agua Amarilla ( $31^{\circ} 06^{\prime} \mathrm{S}, 71^{\circ} 26^{\prime} \mathrm{W}$, 
725 m.s.n.m.), El Huacho ( $31^{\circ} 02^{\prime}$ S, $71^{\circ} 11^{\prime} \mathrm{W}, 775$ m.s.n.m.), La Ciénaga $\left(31^{\circ} 02^{\prime} \mathrm{S}, 71^{\circ} 06^{\prime} \mathrm{W}, 800\right.$ m.s.n.m.), Quebrada Grande ( $31^{\circ} 09^{\prime} \mathrm{S}, 71^{\circ} 16^{\prime} \mathrm{W}, 700$ m.s.n.m.) y, Lomas del Sauce $\left(31^{\circ} 03^{\prime} \mathrm{S}, 71^{\circ} 03^{\prime} \mathrm{W}\right.$, 700 m.s.n.m.) (Figura 1).

Estos sitios están protegidos de la influencia marina directa por una cadena de cerros bajos. El paisaje del área es discontinuo, debido a la existencia de pendientes suaves presentes en sectores planos o casi planos, entremezcladas con lomas bajas interrumpidas por quebradas pequeñas y valles menores (Gastó y Contreras, 1979). Los suelos del área son de textura gruesa y poco desarrollados, aridisoles (Orthids: Camborthids) (Luzio y Alcayaga, 1992). El clima es de tendencia mediterránea subtropical semiárida (Novoa y Villaseca, 1989). La desertificación es elevada en el área de ocurrencia del brote (Gastó y Contreras, 1979; Squeo et al., 2002).

\section{ANTECEDENTES METEOROLÓGICOS DEL ÁREA DE ESTUDIO}

De acuerdo a registros de 30 años, el promedio anual de la temperatura del aire es ca., $16,6^{\circ} \mathrm{C}$ (CV: 19\%, Figura 2). Los meses más fríos son junio y julio $\left(9-12{ }^{\circ} \mathrm{C}\right)$; los más cálidos son enero y febrero $\left(20-22{ }^{\circ} \mathrm{C}\right)$. El promedio de la precipitación total anual es ca., $210 \mathrm{~mm}$, con extremos de 21,8-608,2 mm (CV: 132.0\%) y coeficiente de irregularidad pluviométrica interanual próximo a 3,0 (Figura 3). Mientras la fase árida del sistema dura diez u once meses, comenzando en agosto y terminando en mayo o junio, la fase húmeda se extiende por uno o dos meses, entre mayo y agosto (Figura 4). La variabilidad de los meses con precipitación es también elevada (Figura 5). Los eventos de alta precipitación registrados en el área (e.g., 608,2 y 602,3 mm observados en 1987 y 1997, respectivamente) han sido asociados al fenómeno ENOS (Dillon y Rundel, 1990).

\section{ANTECEDENTES DEMOGRÁFICOS PERÍODO 1970-2000}

La Tabla 1 muestra la ocurrencia de brotes de E. wagenknechti observados durante el período 1970-2000 en el área de Combarbalá. Esta tabla se elaboró sobre la base de denuncias de los agricultores y a los controles efectuados por el Servicio Agrícola y Ganadero, IV Región (Pablo Vera, comunicación personal). En el período registrado, las densidades poblacionales más elevadas correspondieron a fenómenos acaecidos en 1970 y 1977. La densidad poblacional estimada fue de 10-50 $\mathrm{ind} / \mathrm{m}^{2}$ en 1970 (Moroni, 1972; Toro, 1972) y de 15-30 ind $/ \mathrm{m}^{2}$ en 1977 (Pablo Vera, comunicación personal). Estos antecedentes están cercanos a los valores reportados por Pfadt $(1977,1982,1984)$ para ecosistemas norteamericanos de secano y superiores a las estimaciones de densidad encontradas en este trabajo para los brotes de los años 1996 y 1999 (Cepeda-Pizarro et al., en revisión).

Cepeda-Pizarro et al. (en revisión), mediante análisis ANDEVA, determinaron diferencias en densidad entre los brotes de 1996 y $1999\left(\mathrm{~F}_{1,178}\right.$ $=19,50, \mathrm{p}<0,0001$, Tabla 2). Se detectaron diferencias entre sitios para el brote de $1996\left(\mathrm{~F}_{2,87}=\right.$ $3,72 ; \mathrm{p}=0,027)$, pero no para el brote de 1999 $\left(\mathrm{F}_{2,87}=0,19 ; \mathrm{p}=0,824\right)$. Entre brotes, la densidad promedio fue mayor en $1999\left(0,54 \mathrm{ind} / \mathrm{m}^{2} ; 280,6\right.$ $\mathrm{mg} / \mathrm{m}^{2}$ de biomasa seca) respecto de $1996(0,25$ $\mathrm{ind} / \mathrm{m}^{2} ; 130,89 \mathrm{mg} / \mathrm{m}^{2}$ ). El rango de densidad de langostas en el brote de 1996 fluctuó entre 0,17 y $0,37 \mathrm{ind} / \mathrm{m}^{2}$ y entre 88,80 y $191,85 \mathrm{mg} / \mathrm{m}^{2} \mathrm{de}$ biomasa seca, siendo la densidad de la población de Lomas del Sauce significativamente mayor que aquella de los sitios restantes. El rango de densidad de langostas del brote de 1999 fluctuó entre 0,49 y $0,58 \mathrm{ind} / \mathrm{m}^{2}$ y entre 254,85 y $297,80 \mathrm{mg} / \mathrm{m}^{2} \mathrm{de}$ biomasa seca (Tabla 3 ).

\section{RESULTADOS Y DISCUSIÓN}

\section{PATRÓN TÉRMICO}

Aunque la curva de temperatura del aire del período 1994-1996 no difiere en su forma general de la curva histórica, existen algunas diferencias respecto de esta (Figura 7). La temperatura promedio anual del aire del brote de 1996 fue mayor a la temperatura histórica $\left(17,1^{\circ} \mathrm{C}\right.$ y $16,6{ }^{\circ} \mathrm{C}$ respectivamente). Del mismo modo lo fueron las temperaturas promedio de los dos años previos: $18,0^{\circ} \mathrm{C}$ en 1994 y $17,8^{\circ} \mathrm{C}$ en 1995 (Figura 2). También se observó que las temperaturas mínima y máxima del período fueron más altas que sus respectivas históricas. Así, mientras el rango histórico de las temperaturas mínima y máxima del aire fueron ca., $11-21^{\circ} \mathrm{C}$, los rangos observados fueron 13$21{ }^{\circ} \mathrm{C}$ (1996), $12-22{ }^{\circ} \mathrm{C}$ (1995) y $13-22{ }^{\circ} \mathrm{C}$ (1994). 


\section{Cuadro 1}

Registro de 30 años de ocurrencias de eventos irruptivos de $E$. wagenknechti en sitios del secano del norte-centro de Chile ${ }^{1}$

\begin{tabular}{|c|c|c|c|}
\hline año & foco irruptivo & pp anual & fuente alimentaria \\
\hline 1970 & $\begin{array}{l}\text { Quilitapia, Chipel, Divisadero, Lomas del } \\
\text { Sauce, El Huacho, Agua Amarilla, Pama, } \\
\text { Comunidad Jiménez y Tapia, Fundo Las } \\
\text { Añañucas, Quebrada Grande, La Laja } \\
\text { Aeródromo de Chingay, Estación La Mos- } \\
\text { taza, Punitaqui, Camarico Viejo, Ramadilla, } \\
\text { Santa Rosa, El Rodeo, Cerro Las Nipas, } \\
\text { Parral, Alcones. }\end{array}$ & $95,6 \mathrm{~mm}$ & $\begin{array}{l}\text { malvilla, pata de guanaco, dichilla, alfilerillo, } \\
\text { trébol amarillo, pingo-pingo, la reinilla, calandri- } \\
\text { nia, viola. no se reporta ataque a cultivos (trigo, } \\
\text { alfalfa, cebada). }\end{array}$ \\
\hline 1971 & No reportados. & $102,5 \mathrm{~mm}$ & \\
\hline 1972 & $\begin{array}{l}\text { El Huacho, Lomas del Sauce, Quebrada } \\
\text { Trujillo, Pama, Chingay, Quilitapia. }\end{array}$ & $30,9 \mathrm{~mm}$ & $\begin{array}{l}\text { vegetación xerófila (pichanilla, incienso, pasto } \\
\text { salado ataca cultivos (trigo, porotos, cebolla, alfalfa, } \\
\text { habas y partes bajas de perales y vid). }\end{array}$ \\
\hline 1973 & No reportados. & & \\
\hline 1974 & $\begin{array}{l}\text { Chipel, Quebrada de Lepe, Divisadero, El } \\
\text { Huacho, Lomas del Sauce Agua Amarilla, } \\
\text { Comunidad Jiménez y Tapia, Quilitapia, } \\
\text { Quebrada Grande, Pajarito Blanco, La Cié- } \\
\text { naga, Cancha de aterrizaje de Quilitapia, } \\
\text { Peña Blanca y Chingay. }\end{array}$ & $107,5 \mathrm{~mm}$ & vegetación nativa. Trigo no afectado. \\
\hline 1975 & $\begin{array}{l}\text { Trujillo, Chipel, Lepe, Peñablanca, } \\
\text { El Carrizo, Los Romeros, Sauce Bajo, Agua } \\
\text { Amarilla, Quebrada de Lepe. }\end{array}$ & $118,1 \mathrm{~mm}$ & $\begin{array}{l}\text { vegetación nativa. Cultivos levemente afectados } \\
\text { (trigo, alfalfa, comino, arvejas, hortalizas). }\end{array}$ \\
\hline 1976 & No reportados. & $125 \mathrm{~mm}$ & \\
\hline 1977 & $\begin{array}{l}\text { Sectores Altos de las Cañuelas de Quilita- } \\
\text { pia, Agua Amarilla, Cancha de aterrizaje } \\
\text { de Quilitapia, Andacollito del Huacho, } \\
\text { Majada de la Morra, Cruz Grande del } \\
\text { Huacho, Quebrada de Trujillo, Divisadero, } \\
\text { Llanos de Bucho, Pama, Chingay, Soruco, } \\
\text { Lepe, Cañuelas de Peña Blanca, Quebrada } \\
\text { del Huacho. }\end{array}$ & $76 \mathrm{~mm}$ & $\begin{array}{l}\text { vegetación nativa. Cultivos levemente afectados } \\
\text { (cereales, frutales). }\end{array}$ \\
\hline $1978-79$ & No reportado. & $113,5 / 27 \mathrm{~mm}$ & \\
\hline 1980 & $\begin{array}{l}\text { Quilitapia, Las Quebradas, El Huacho, El } \\
\text { Pacul, Lomas del Sauce, Lepe, Soruco, } \\
\text { Cogotí, Pama, Pama Arriba, Blanquillo, } \\
\text { Chingay, Placa (Combarbalá). }\end{array}$ & $46 \mathrm{~mm}$ & vegetación nativa. Cultivos levemente afectados. \\
\hline $1981-95$ & No reportado. & & \\
\hline 1996 & $\begin{array}{l}\text { Agua Amarilla, El Huacho, Lomas del } \\
\text { Sauce, Pajarito Blanco, La Ciénaga, } \\
\text { Quilitapia. }\end{array}$ & $120 \mathrm{~mm}$ & vegetación nativa. Cultivos levemente afectados. \\
\hline $1997-98$ & No reportado. & $608,2 / 20 \mathrm{~mm}$ & \\
\hline 1999 & $\begin{array}{l}\text { Lomas del Sauce, Agua Amarilla, } \\
\text { El Huacho. }\end{array}$ & $165,8 \mathrm{~mm}$ & vegetación nativa. Cultivos levemente afectados. \\
\hline 2000 & No reportado. & $198,2 \mathrm{~mm}$ & \\
\hline
\end{tabular}

${ }^{1}$ Fuente de la información: Servicio Agrícola Ganadero (IV Región, Coquimbo).

${ }^{1}$ Source of information: Agricultural and Livestock Service (Region IV, Coquimbo). 
Cuadro 2

Valores del ANDEVA para efecto de sitio y año del evento sobre la densidad $\left(\mathrm{N}^{\circ} \mathrm{ind} / \mathrm{m}^{2}\right) \mathrm{de}$ E. wagenknechti (Orthoptera: Tristiridae) en dos eventos de irrupción poblacional ocurridos en sitios de secano de Chile nor-central

\begin{tabular}{clrcccc}
\hline Evento & \multicolumn{1}{c}{ FV } & gl & SC & MC & F & p (F) \\
\hline \multirow{2}{*}{1996} & entre sitio & 2 & 0,661 & 0,330 & 3,72 & 0,0276 \\
& error & 87 & 7,739 & 0,088 & & \\
\multirow{2}{*}{1999} & entre sitio & 2 & 0,116 & 0,058 & 0,19 & 0,8243 \\
& error & 87 & 26,205 & 0,301 & & $<0,0001$ \\
\multirow{2}{*}{$1996 / 99$} & entre eventos & 1 & 3,803 & 3,803 & 19,50 & \\
& error & 178 & 34,723 & 0,195 & & \\
\hline
\end{tabular}

Cuadro 3

Densidad promedio de $E$. wagenknechti en dos eventos de irrupción poblacional ocurridos en sitios de secano de Chile nor-central

\begin{tabular}{|c|c|c|c|c|}
\hline Evento & sitio & ind $/ \mathbf{m}^{2} \pm$ ee & ind/ha & $\mathrm{mg} / \mathrm{m}^{2} \pm$ ee1 \\
\hline \multirow[t]{4}{*}{1996} & Lomas del Sauce & $0,37 \pm 0,07 \mathrm{a}$ & 3.700 & $191,85 \pm 35,8 \mathrm{a}$ \\
\hline & La Ciénaga & $0,22 \pm 0,03 b$ & 2.200 & $111,68 \pm 15,4 b$ \\
\hline & Quebrada Grande & $0,17 \pm 0,06 \mathrm{~b}$ & 1.700 & $88,80 \pm 28,9 b$ \\
\hline & Promedio evento & $0,25 \pm 0,05 \mathrm{a}$ & 2.500 & $130,89 \pm 26,7 \mathrm{a}$ \\
\hline \multirow[t]{4}{*}{1999} & Lomas del Sauce & $0,56 \pm 0,10 \mathrm{a}$ & 5.600 & $289,21 \pm 52,5 \mathrm{a}$ \\
\hline & Agua Amarilla & $0,58 \pm 0,10 \mathrm{a}$ & 5.800 & $297,80 \pm 52,0 \mathrm{a}$ \\
\hline & El Huacho & $0,49 \pm 0,10 \mathrm{a}$ & 4.900 & $254,85 \pm 50,3 \mathrm{a}$ \\
\hline & Promedio evento & $0,54 \pm 0,10 \mathrm{~b}$ & 5.400 & $280,6 \pm 51,6 b$ \\
\hline
\end{tabular}

1 Dada la marcada diferencia de tamaño entre los sexos, la biomasa se expresa como el peso seco promediado entre los pesos secos del macho y de la hembra (137,7 y 423,6 mg, respectivamente). Valores seguidos por una misma letra no son estadísticamente significativos (prueba de $\mathrm{LSD}, \mathrm{p}<0,05$ ). $\mathrm{n}=30$ cuadrantes de $12 \times 1,2 \mathrm{~m}$ por sitio.

1 Given the strong difference of body size between the sexes, the biomass is expressed as the averaged dry weight between the male and female dry weight (137.7 and $423.6 \mathrm{mg}$ respectively). Values followed by the same letter are not statistically significant (LSD-test, $\mathrm{p}<0.05$ ). $\mathrm{n}=30$ quadrats of $12 \times 1.2 \mathrm{~m}$ by site.

Por otro lado, mientras la temperatura máxima del área $\left(\mathrm{ca} ., 21^{\circ} \mathrm{C}\right)$ se alcanzó en enero-febrero tanto en la curva histórica como en los años 1996 y 1995. Esta temperatura máxima se logró en enero de 1994 (Figura 6). Las curvas de variabilidad térmica mensual del período 1997-1999 también mostraron una figura similar a la curva histórica, pero con valores mínimos y máximos ligeramente más altos que los valores históricos (Figura 7).

\section{PATRÓN PLUVIOMÉTRICO}

La distribución de la precipitación mensual registrada en 1994 fue similar al patrón histórico, no así las distribuciones de los años 1995 y 1996 (Figura 8). La distribución de 1995 fue similar respecto de la distribución histórica y a un evento de precipitación en enero el cual aportó $10 \mathrm{~mm}$ de agua, comparado al promedio histórico de ca., $0 \mathrm{~mm}$. 


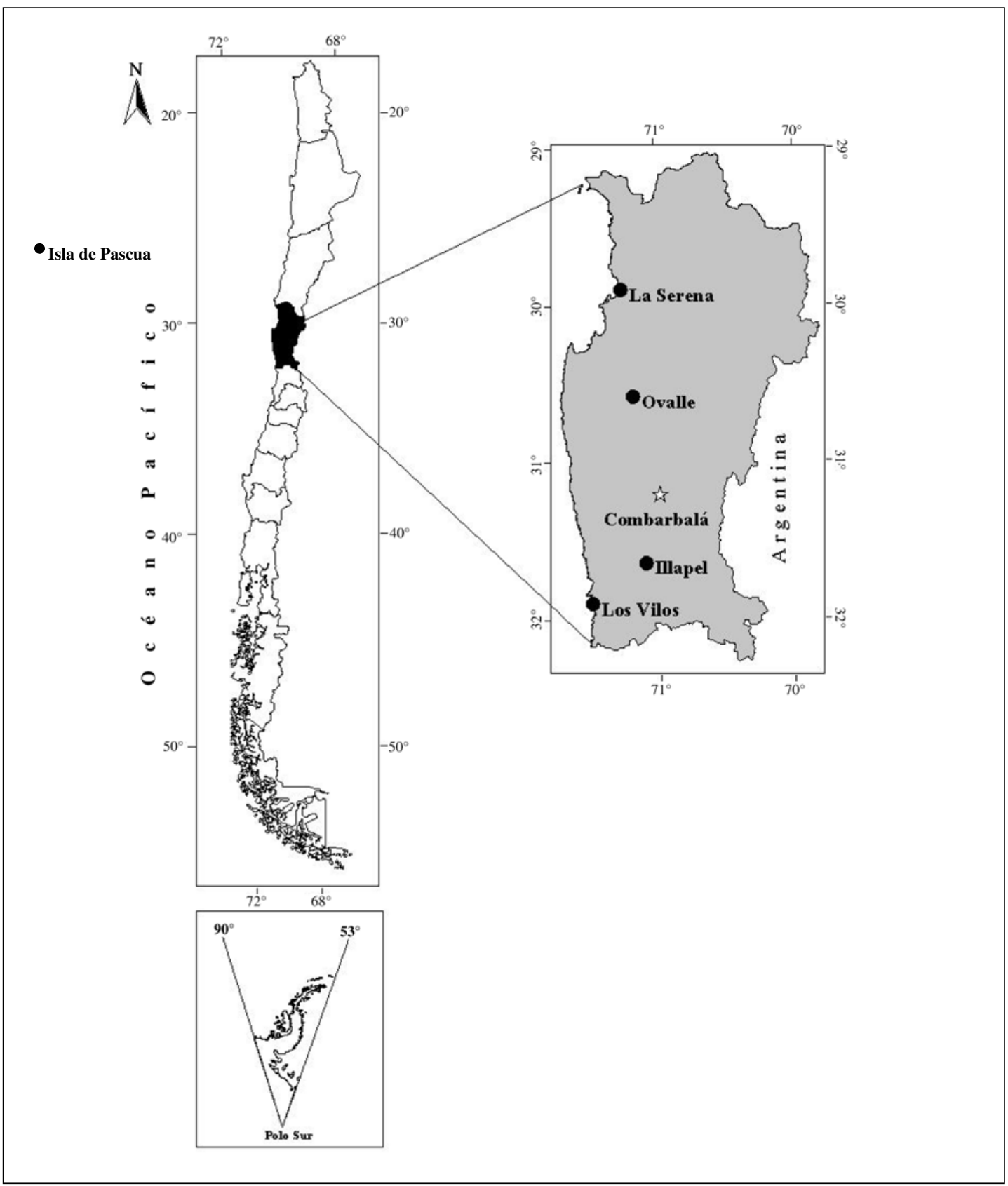

Figura 1. Ubicación del área de estudio (Combarbalá, $31^{\circ} 10^{\prime} \mathrm{S}, 71^{\circ} 00^{\prime} \mathrm{O}$ ) en el norte-centro de Chile.

La distribución de 1996 mostró un evento lluvioso en abril el cual aportó $c a$., $18 \mathrm{~mm}$ de agua, comparado al valor esperado de $c a$., $12 \mathrm{~mm}$ (Figura 8).

La precipitación total anual medida en 1970 fue $c a$., $96 \mathrm{~mm}$ (54\% de déficit), distribuyéndose en los meses de mayo (31\%), julio (50\%) y octubre $(19 \%)$. En los dos años previos al brote, la precipi- tación observada fue ca., $40 \mathrm{~mm}$ ( $81 \%$ de déficit) en 1969, y ca., $60 \mathrm{~mm}$ (71\% de déficit) en 1968 . Según muestra la Figura 6, además del déficit en precipitación registrado en los tres años, existió una alteración en la forma general del patrón mensual de distribución de ella, particularmente en los dos años previos al brote. Por ejemplo, en 1969 se 


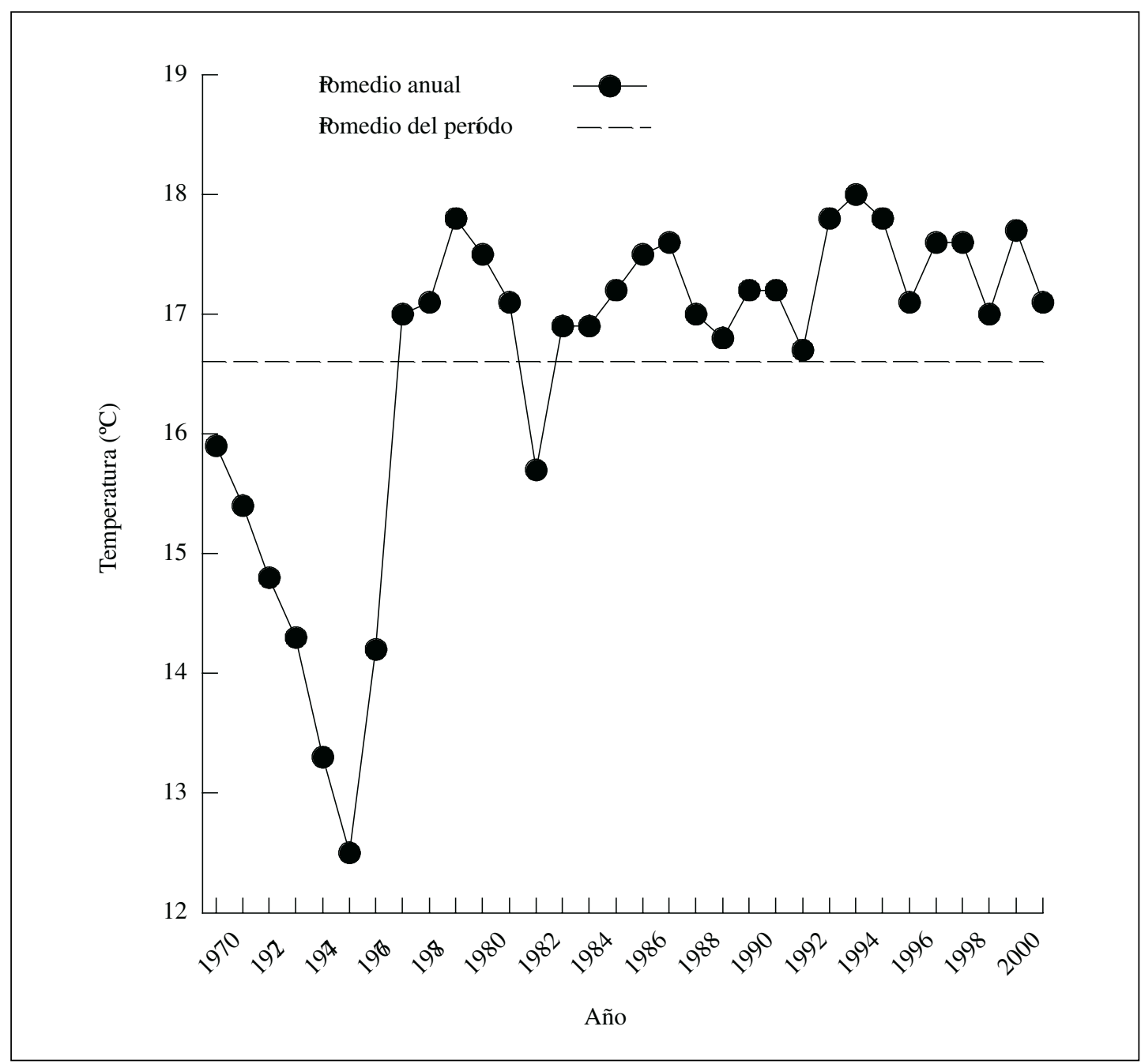

Figura 2. Variabilidad de la temperatura promedio anual del aire $\left({ }^{\circ} \mathrm{C}\right)$ durante el período 1970-2001, y el promedio de 30 años (----) registrados en el área de Combarbalá (Estación Meteorológica Embalse Cogotí, 3102’S, 71º8’O).

distribuyó en mayo-junio (55\%) y en agosto (45\%); en 1968 , se concentró en los meses de junio $(75 \%)$ y septiembre $(25 \%)$.

El año del brote de 1996 tuvo una precipitación total anual de ca., $110 \mathrm{~mm}$ (48\% de déficit). Fue precedido por dos años secos: $70 \mathrm{~mm}$ en 1995 (67\% de déficit) y 72 mm (66\% de déficit) en 1994 (Figura 3). La distribución de la precipitación mensual del año 1994 fue muy similar al patrón histórico, no así las distribuciones de los años 1995 y 1996 (Figura 8).

El brote poblacional de 1999 tuvo asociado dos fenómenos pluviométricos importantes. En primer lugar, el año 1998 contribuyó con el valor más bajo de la serie de datos de precipitación registrada hasta el momento en el área de Combarbalá (e.g., 21,8 mm). En segundo lugar, el año 1997 aportó el segundo valor más alto de precipitación (e.g., 602,3 mm) (Figura 3). En general, se repitió el patrón térmico asociado al brote de 1996. La temperatura promedio anual del aire fue ligeramente superior al valor histórico, $17{ }^{\circ} \mathrm{C}$ y $16,6^{\circ} \mathrm{C}$, respectivamente. El brote estuvo precedido por dos años con temperaturas sobre el promedio, $17{ }^{\circ} \mathrm{C}$ en 1998 y $17.6^{\circ} \mathrm{C}$ en 1997 (Figura 2). La precipitación anual registrada durante el brote del año 1999 tuvo un déficit del $29 \%$ en comparación al promedio (150mm y $210 \mathrm{~mm}$, respectivamente). El brote estuvo precedido por un año 


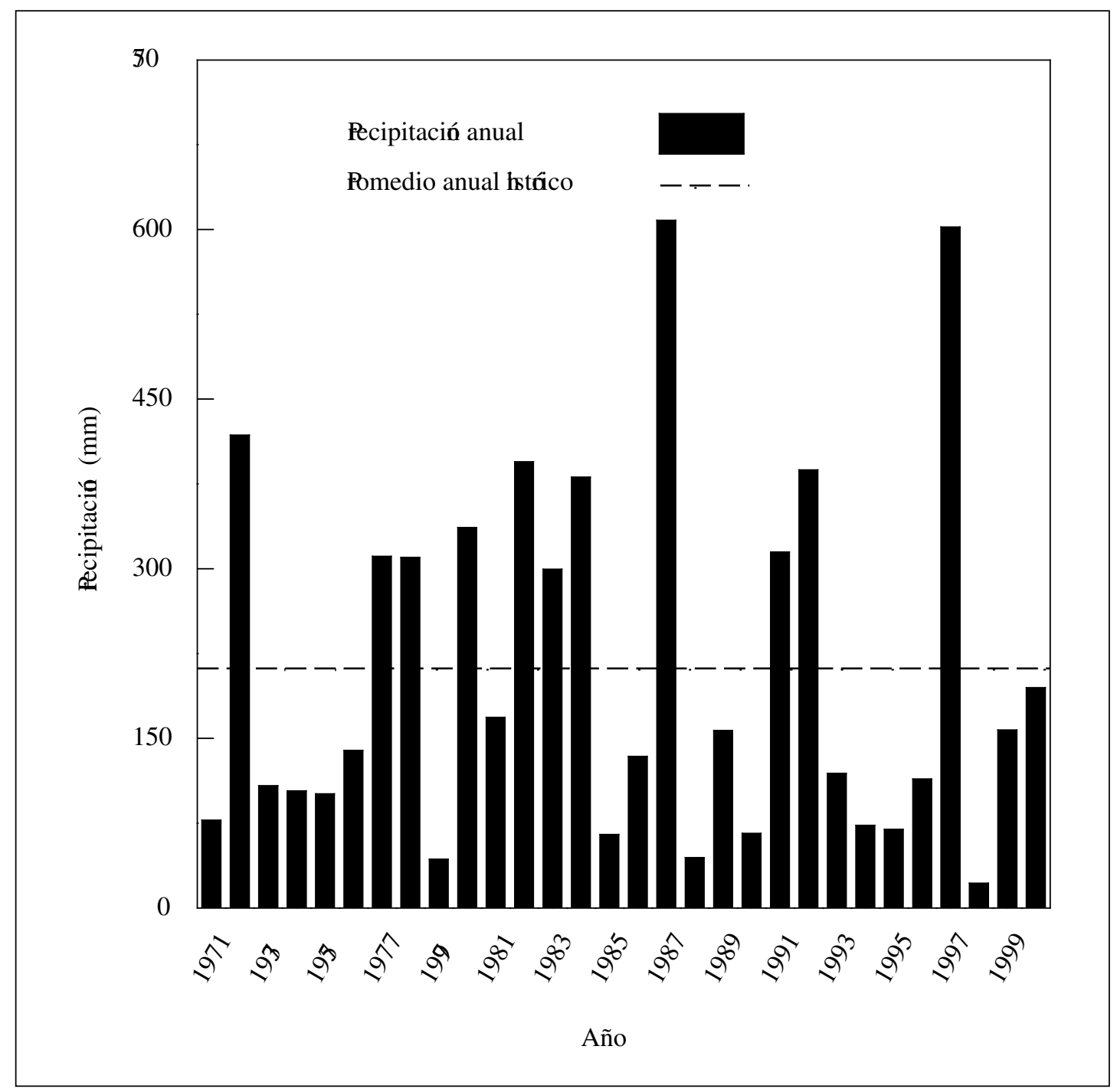

Figura 3. Variabilidad de la precipitación total anual (mm) durante el período 1971-2000 y registrada en el área del Combarbalá. La línea horizontal (----) representa el promedio anual histórico (Estación Meteorológica Combarbalá, 31º' $10^{\prime} \mathrm{S}, 71^{\circ} 00^{\prime} \mathrm{O}$ ).

muy seco (90\% de déficit de precipitación en 1998) y por un año extremadamente lluvioso (187\% de superávit en 1997). Las curvas de distribución mensual de la precipitación presentan ligeras asimetrías respecto de la curva histórica (Figura 8). En 1999, por ejemplo, el período de máxima precipitación se desplazó en un mes hacia el término del invierno y él ca., $57 \%$ de ésta se concentró en agosto-octubre, con un superávit para el período de $224 \%$ respecto del aporte histórico de ese período. La asimetría del año 1998 fue evidente, con una pequeña cantidad de agua caída en los meses de mayo y junio. La distribución mensual de la precipitación del año 1997 mostró elevados valores de aporte pluvial en junio (607\% sobre el promedio del mes), agosto (581\% sobre el promedio) y octubre (786\% sobre el promedio).

\section{CONCLUSIONES}

Los antecedentes que disponemos a la fecha no permiten establecer cuales son las características de estos hábitats que están determinando su 


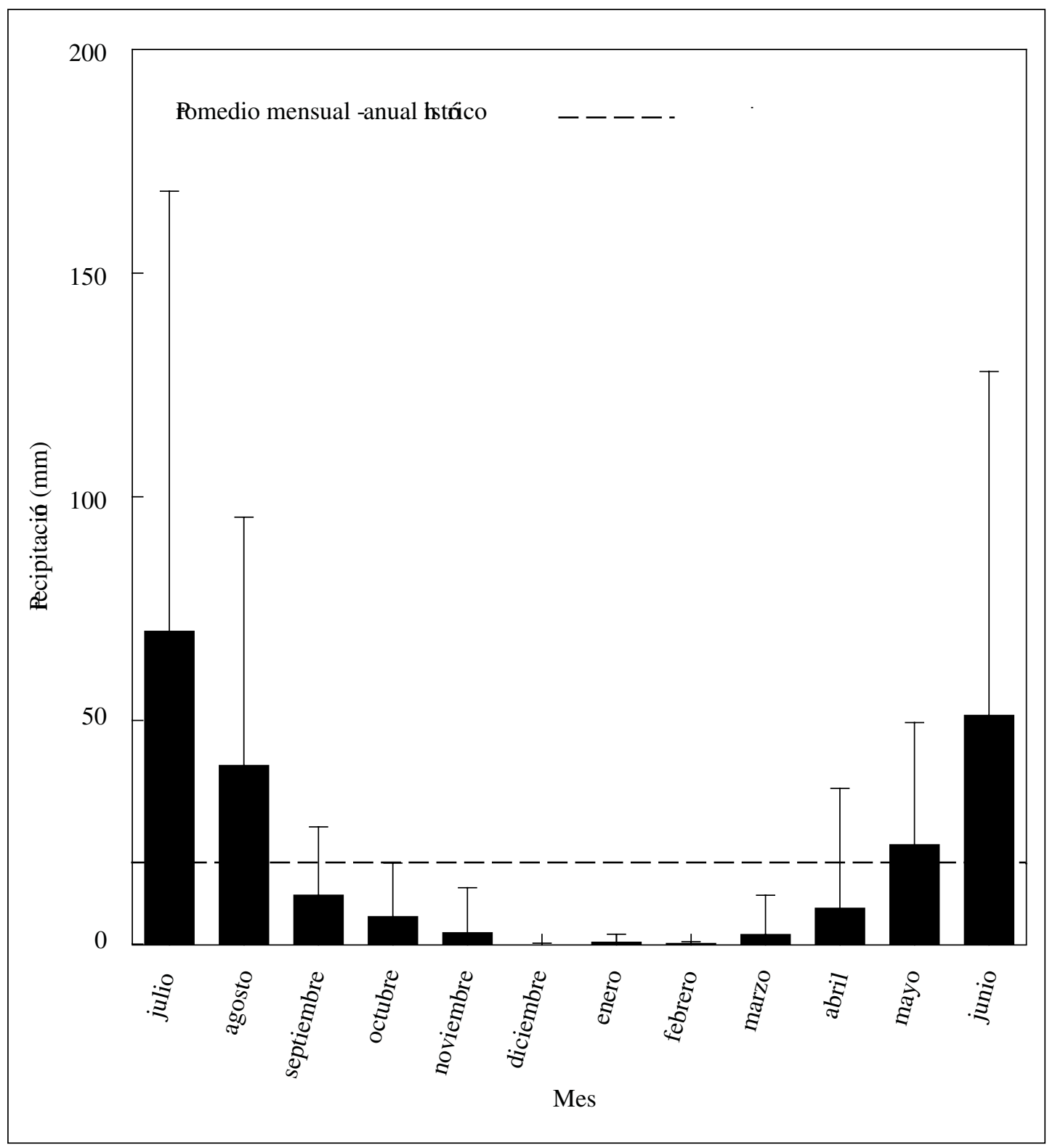

Figura 4. Variabilidad de la precipitación mensual promedio $(\mathrm{mm})$ registrada en el área del Combarbalá durante los últimos 30 años. La línea horizontal (----) representa el promedio mensual-anual histórico (30 años) de la precipitación (Estación Meteorológica Combarbalá, $\left.31^{\circ} 10^{\prime} \mathrm{S}, 71^{\circ} 00^{\prime} \mathrm{O}\right)$.

condición de foco irruptivo. No obstante lo anterior, trabajos existentes en la literatura respecto de la dinámica poblacional de acrídidos de ecosistemas áridos consignan el papel que pueden cumplir tanto las características del hábitat como su estado de alteración (Fielding y Brusven, 1993, 2001; Campbell, 2001; Vermeire et al., 2004). Creemos que el laboreo agrícola en los cultivos de secano y el posterior abandono de las tierras puede estar contribuyendo a ello.

El análisis de la variabilidad térmica y pluviométrica registrada en los años de los brotes de $E$. wagenknechti en el área reveló ciertas alteraciones respecto de los patrones históricos mostrados por estos factores ambientales. En primer lugar y desde el punto de vista meteorológico, la zona 


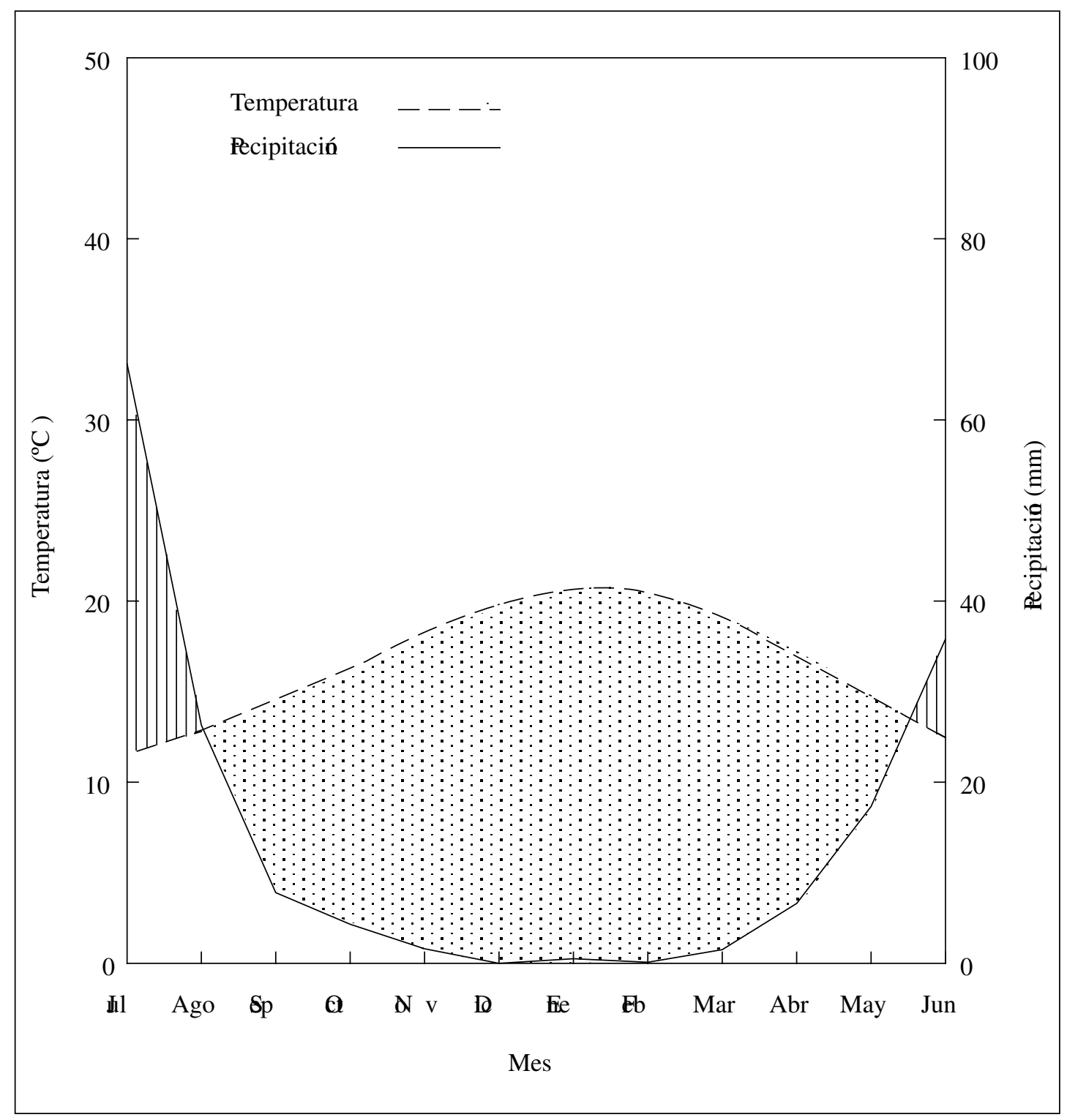

Figura 5. Climodiagrama del área de estudio.

del estudio se caracteriza por una temperatura del aire relativamente constante $\left(\mathrm{ca} ., 16,6^{\circ} \mathrm{C}\right.$ promedio anual). Posee una fase árida que dura 10 meses. La precipitación tiene una elevada variabilidad interanual, promedia los $c a$., $210 \mathrm{~mm}$ y ocurre concentrada en los meses de junio y julio. El primer brote de E. wagenknechti registrado en la literatura científica tuvo lugar en 1970. La temperatura promedio anual de aire de ese año fue cercana al promedio histórico $\left(\mathrm{ca} ., 16^{\circ} \mathrm{C} \mathrm{y}\right.$ $16,6{ }^{\circ} \mathrm{C}$, respectivamente).
Aun cuando los resultados encontrados en este trabajo no son concluyentes respecto de la importancia de la temperatura y la precipitación en la determinación de los brotes E. wagenknechti en el área de Combarbalá, aparentemente la combinación de temperaturas sobre el promedio en los años previos al brote -acompañadas por años secos precedidos por años con lluvia sobre un cierto umbral- estaría gatillando la emergencia de este fenómeno. Del mismo modo, el patrón de distribución mensual de la precipitación podría estar jugando un papel fundamental. 


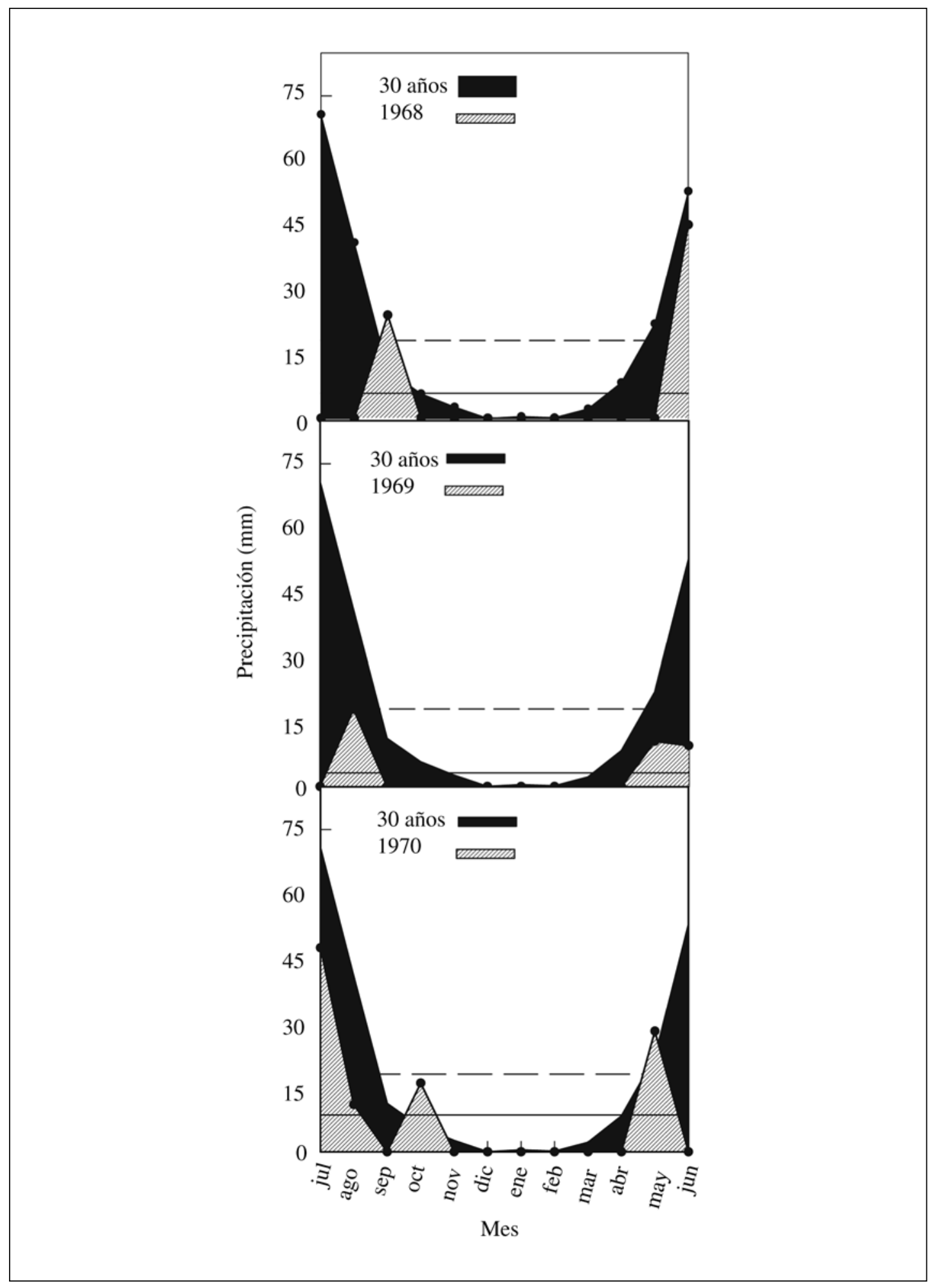

Figura 6. Distribución de la precipitación mensual registrada durante el período 1968-1970. Sobre esta distribución se ha sobrepuesto la distribución anual-mensual histórica. La línea discontinua horizontal representa el promedio mensual-anual histórico de

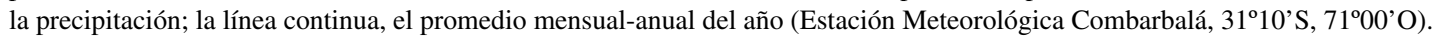




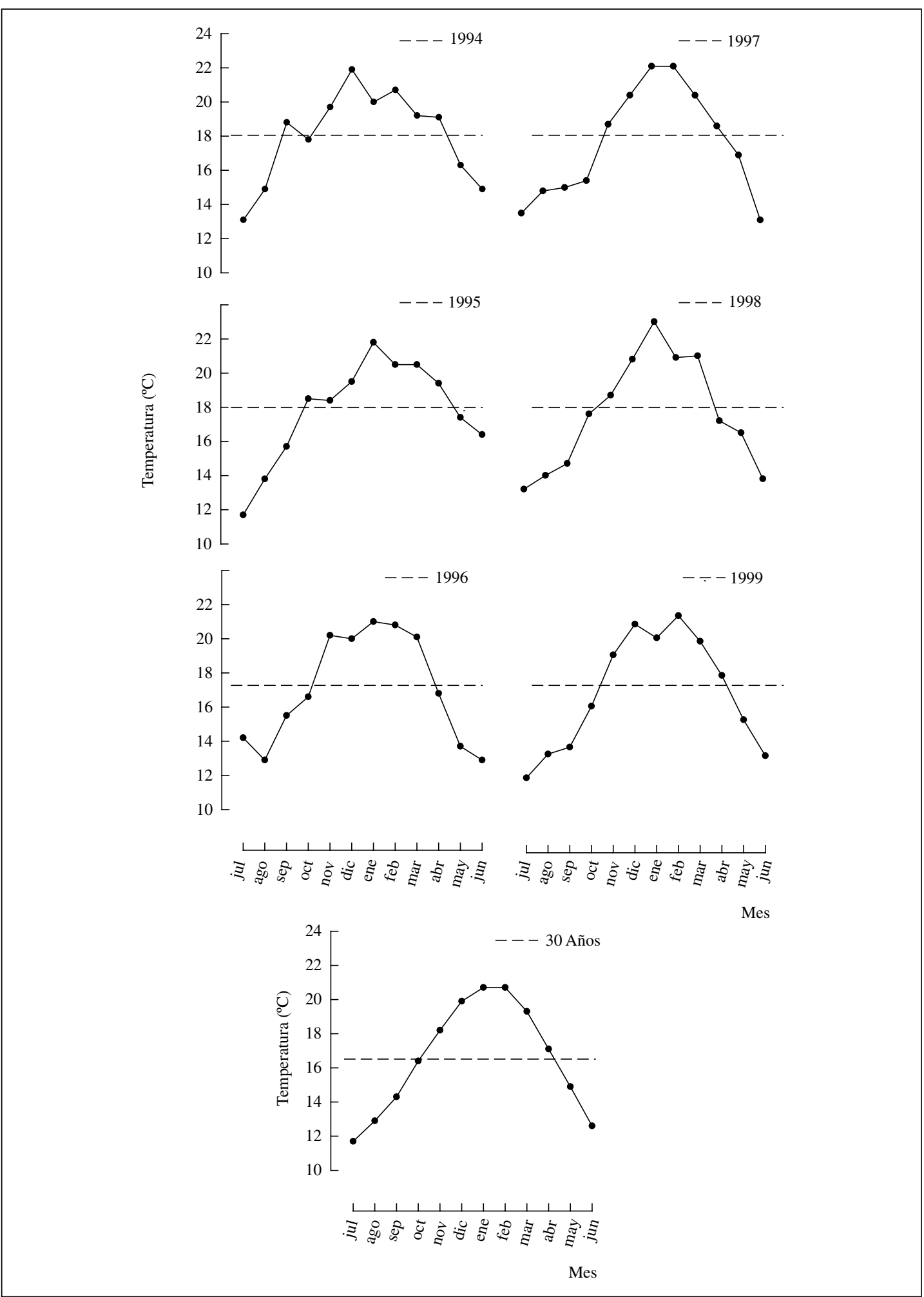

Figura 7. Distribución de la temperatura mensual promedio del aire $\left({ }^{\circ} \mathrm{C}\right)$ del período $1994-1999$ y del promedio histórico $(30$ años) registrada en el área de Combarbalá. La línea horizontal discontinua representa el valor promedio anual del año o del período

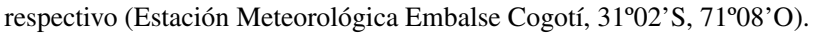




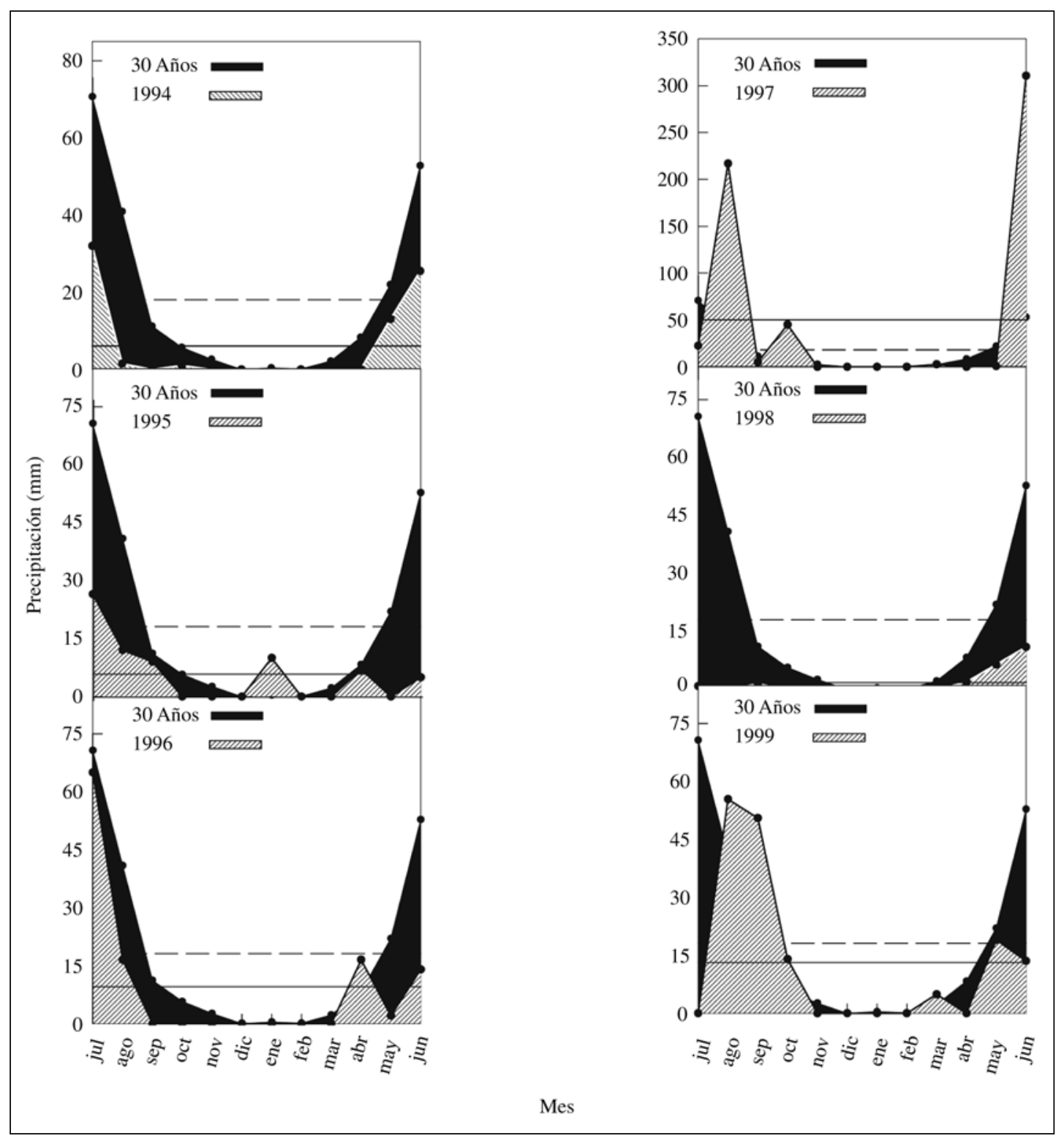

Figura 8. Distribución de la precipitación mensual registrada durante el período 1994-1999. Sobre esta distribución se ha superpuesto la distribución anual-mensual histórica. La línea cortada horizontal representa el promedio mensual-anual histórico de la

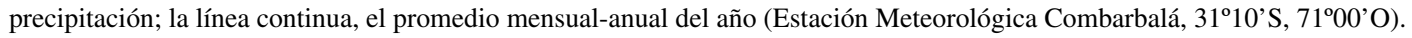

\section{RECONOCIMIENTOS}

Los autores agradecen a Julio R. Gutiérrez (Universidad de La Serena) y Pablo Vera (SAG IV Región) por los comentarios y sugerencias realizados a la versión anterior del manuscrito, y a David López Aspe (Centro de Estudios Avanzados en Zonas Áridas (CEAZA), La Serena, Chile) por la asistencia en SIG.

\section{LITERATURA CITADA}

AMARASEKARE, K.G.; J.V. EDELSON (2004). Effect of temperature on efficacy of insecticides to differential grasshopper (Orthoptera: Acrididae). Journal of Economic Entomology 97: 1595-1602.

BAUER, S.; J. SAMIETZ; U. BERGER (2005). Sexual harassment in heterogeneous landscapes can mediate population regulation in a grasshopper. Behavioral Ecology 16: 39-246. 
BERNER, D.; C. KORNER; W. U. BLANCKENHORN (2004). Grasshopper populations across $2000 \mathrm{~m}$ of altitude: is there life history adaptation?. Ecography. 27: 733-740.

CAMPBELL, J.B. (2001). Anti-quality effects of insects feeding on rangeland plants: A review. Journal of Range Management 54: 462-465.

CAPINERA, J.L.; D.R. HORTON (1989). Geographic variation in effects of weather on grasshopper infestation. Environmental Entomology 18: 8-14.

CEPEDA-PIZARRO, J.; S. VEGA; H. VÁSQUEZ; M. ELGUETA (2003). Morfometría y dimorfismo sexual de Elasmoderus wagenknechti (Liebermann) (Orthoptera: Tristiridae) en dos eventos de irrupción poblacional. Revista Chilena de Historia Natural 76: 417-435.

CEPEDA-PIZARRO, J.; S. VEGA; H. VÁSQUEZ; M. ELGUETA; J. PIZARRO-ARAYA. Demography of two populations outbreaks of Elasmoderus wagenknechti (Orthoptera: Tristiridae) in the semiarid region of Chile. Neotropical Entomology (en revisión).

CIGLIANO, M. M. (1989a). Revisión sistemática de la familia Tristiridae (Insecta: Orthoptera). Boletín de la Sociedad de Biología de Concepción (Chile) 60: 89-91.

CIGLIANO, M. M. (1989b). A cladistics analysis of the family Tristiridae (Orthoptera, Acridoidea). Cladistics 5: 379-393.

CIGLIANO, M. M. (1991). Congruencia taxonómica en la familia Tristiridae (Orthoptera, Acridoidea). Revista de la Sociedad Entomológica Argentina 49: 85-101.

CIGLIANO, M. M.; R. A. RONDEROS; W. P. KEMP (1989). Revision of the genus Elasmoderus Saussure (Orthoptera: Tristiridae). Canadian Entomologist 121: 225-243.

CORCKET, E.; I. CHINTAUAN-MARQUIER; R. M. CALLAWAY; R. MICHALET (2002). Species-dependence and environmental changes in grasshoppers herbivory. Comptes Rendus Biologies 325: 155-164.

DILLON, M. O.; P. W. RUNDEL (1990). The botanical response of the Atacama and Peruvian Desert flora to the 1982-83 El Niño Event. p. 487-504. In P.W. Glynn (ed.) Global Ecological Consequences of the 1982-83 El NiñoSouthern Oscillation. Elsevier Oceanographic Series 52: Amsterdam, The Netherlands.

DONATO, M. (2006). Historical biogeography of the family Tristiridae (Orthoptera: Acridomorpha) applying dispersal-vicariance analysis. Journal of Arid Environments 66: 421-434.

FARROW, R. A.; D. A. LONGSTAFF (1986). Comparison of the annual rates of increase of locusts in relation to the incidence of plagues. Oikos 46: 207-222.

FIELDING, D. J.; M. A. BRUSVEN (1990). Historical analysis of grasshopper (Orthoptera: Acrididae) population responses to climate in southern Idaho. Environmental Entomology 19: 1786-1791

FIELDING, D.J.; M. A. BRUSVEN (1993). Spatial analysis of grasshoppers density and ecological disturbance on southern Idaho rangeland. Agriculture, Ecosystems \& Environment 43: 31-47.

FIELDING, D. J.; M. A. BRUSVEN; B. SHAFII; W. J. PRICE (2001). Spatial heterogeneity of low-density populations of Melanoplus sanguinipes (Orthoptera: Acrididae) associated with grazing and vegetation treatments. Canadian Entomologist 133: 843-855.
GAGE, S. H.; M. K. MUKERJI (1977). A perspective of grasshopper population distribution in Saskatchewan and the interrelationship with weather. Environmental Entomology 6: 469-479.

GARDNER, K. T.; D.C. THOMPSON (2001). Development and phenology of the beneficial grasshopper Hesperotettix viridis (Orthoptera: Acrididae). Southwestern Entomologist 26: 305-313.

GASTÓ, J.; D. CONTRERAS (1979). Un caso de desertificación en el norte de Chile. 99 p. El ecosistema y su fitocenosis. Bol. Téc. $\mathrm{N}^{\circ}$ 42. Facultad de Agronomía. Universidad de Chile. Santiago, Chile.

JOERN, A.; S. B. GAINES (1990). Populations dynamics and regulation in grasshoppers. p. 415-482. In R.F. Chapman and A. Joern (eds.) Biology of grasshoppers. John Wiley and Sons. New York, USA.

KEMP, W. P.; M. M. CIGLIANO (1994). Drought and rangeland grasshopper species diversity. Canadian Entomologist 126: 1075-1092.

LOCKWOOD, J.A.; D. R. LOCKWOOD (1991). Rangeland grasshopper (Orthoptera: Acrididae) population dynamics: insight from catastrophe theory. Environmental Entomology 20: 970-980.

LUKER, L. A.; J. D. HATLE; S. A. JULIANO (2002). Reproductive responses to photoperiod by a south Florida population of the grasshopper Romalea microptera (Orthoptera: Romaleidae). Environmental Entomology 31: 702-707.

LUZIO, W.; S. ALCAYAGA (1992). Mapa de asociaciones de grandes grupos de suelos de Chile. Agricultura Técnica 52: 347-353.

MORONI, J. (1972). Irrupción de Elasmoderus rabiosus (Liebermann) en el Norte Chico (Acrididae, Chilacridinae). Noticiero Mensual del Museo Nacional de Historia Natural (Chile) 192: 3-6.

MORRONE, J. J. (2006). Biogeographic areas and transition zones of Latin America and the Caribbean islands based on Panbiogeographic and cladistic analyses of the entomofauna. Annual Review of Entomology 51: 467-494.

NERNEY, N. J. (1961). Effects of seasonal rainfall on range condition and grasshopper population, San Carlos Apache Indian Reservation, Arizona. Journal of Econnomic Entomology 54: 382-385.

NOVOA, R.; Y. S. VILLASECA (1989). Mapa agroclimático de Chile. 221 p. INIA. Santiago, Chile.

PFADT, R. E. (1977). Some aspects of the ecology of grasshopper populations inhabiting the short grass plains. p. 73-79. In: H.M. Kulman and H.C. Chiang (eds.) Insect Ecology. University of Minnesota Agricultural Experimental Station, Technical Bulletin 310, Minnesota, USA.

PFADT, R. E. (1982). Density and diversity of grasshoppers (Orthoptera: Acrididae) in an outbreak on Arizona rangeland. Environmental Entomology 11: 690-694.

PFADT, R. E. (1984). Species richness, density, and diversity of grasshoppers (Orthoptera: Acrididae) in a habitat of the mixed grass prairie. Canadian Entomologist 116: 703-709.

ROIG-JUÑENT, S.; G. E. FLORES (2001). Historia biogeográfica de las áreas áridas de América del Sur Austral. p. 257-266. In: J. Llorente Busquets y J.J. Morrone (eds.) Introducción a la biogeografía en Latinoamérica: Teorías, conceptos, métodos y aplicaciones. Las prensas de Ciencias, Facultad de Ciencias, UNAM, México, D.F. 
ROIG-JUNENT, S.; M. C. DOMÍNGUEZ; G. E. FLORES; C. MATTONI (2006). Biogeographic history of South American arid lands: A view from its arthropods using TASS analysis. Journal of Arid Environments 66: 404-420.

SCHELL, S. P.; J. A. LOCKWOOD (1997). Spatial analysis of ecological factors related to rangeland grasshopper (Orthoptera: Acrididae) outbreaks in Wyoming. Environmental Entomology 26: 1343-1353.

SKINNER, K. M.; R. D. CHILD (2000). Multivariate analysis of the factors influencing changes in Colorado grasshopper abundance. Journal of Orthoptera Research 9: 103-109.

SQUEO, F. A.; G. ARANCIO; C. MARTICORENA; M. MUÑOZ; J. R. GUTIÉRREZ (2002). Diversidad vegetal en la IV Región de Coquimbo, Chile. p. 149-158. In F.A. Squeo, G. Arancio y J.R. Gutiérrez (eds.), Libro rojo de la flora nativa y de los sitios prioritarios para su conservación: Región de Coquimbo. Ediciones Universidad de La Serena. La Serena. Chile.

TORO, H. (1972). Notas biológicas sobre la langosta de Combarbalá. Idesia (Chile) 2: 133-136.

VERMEIRE, L. T.; R. B. MITCHELL; S. D. FUHLENDORF; D. B. WESTER (2004). Selective control of rangeland grasshoppers with prescribed fire. Journal of Range Management 57: 29-33.

WACHTER, D. H.; K. M. O'NEILL; W. P. KEMP (1998). Grasshopper (Orthoptera: Acrididae) communities on an elevational gradient in southwestern Montana. Journal of the Kansas Entomological Society 71: 35-43.

WAGNER, W. E. (2005). Male field crickets that provide reproductive benefits to females incur higher costs. Ecological Entomology 30: 350-357. 